\title{
Infrared Control of Thermal Insulation of Teeth, Dental Crowns and Braces When Inhaling Cold Air with an Open Mouth in the Frosty Day
}

\author{
by A. Urakov*, M. Alies*, A. Reshetnikov*, M. Kopylov*, R. Rozov**, \\ *Udmurt Federal Research Center of the Ural branch of the Russian Academy of Sciences, Department of \\ Modeling and Synthesis of Technological Processes, T. Baramzina str., 34, 426067, Izhevsk, Russia, email of \\ corresponding author: urakoval@live.ru; \\ **.P.Pavlov First St.-Petersburg State Medical University, Department of Prosthetic Dentistry, Leo Tolstoy \\ str., 6-8, 197022, St.-Petersburg, Russia.
}

\begin{abstract}
It is shown that in severe cold, the performance of intensive physical activity is accompanied by intensive inhalation of frosty air with an open mouth, which cools the teeth. Sometimes the frosty air destroys teeth. Especially quickly and strongly cooled metal crowns, dentures and braces. To protect the teeth and soft tissues of the oral cavity from hypothermia and frostbite, it was proposed to cover the teeth with special decorative stickers. It is shown that thermal monitoring of the local temperature of teeth in the cold provides an assessment of the effectiveness of thermal insulation of teeth in real time.
\end{abstract}

\section{Introduction}

Biathletes, skaters, mountaineers, mountain rescuers, military personnel under intense physical exertion in the cold in the highlands are forced to breathe with an open mouth. This quickly cools the teeth and gums, which can damage their enamel. In recent years, metal dentures and braces have been widely installed in dentistry, which themselves can cause damage to the cheeks and lips [1,2]. To detect their local damaging effects, it is recommended to use a thermal imager. However, the diagnostic value of thermal imaging monitoring and the pathological value of these dental structures for cold injuries of the oral cavity when inhaling frosty air remains unexplored.To protect the teeth from damage in the cold was invented "Decorative sticker for tooth heat insulation" (RU 2698349) [3].

\section{Result and discussion}

We studied the dynamics of the local temperature of the front surface of the teeth in adult healthy volunteers when inhaling cold air with an open mouth for 10 minutes at an air temperature of $-10^{\circ} \mathrm{C}$. the first group included volunteers without dental crowns, prostheses and braces. The second group included volunteers with metal braces installed a few months ago. The third group included volunteers with metal dental crowns, which were installed by them a few years ago. The local temperature in the hard and soft tissues of the oral cavity before, during and after inhaling cold air was measured using a thermal imager in all the subjects.

The subjects in the all groups were divided into two subgroups. In one subgroup there were people with open dentition and/or dental structures, in another subgroup there were people with teeth and/or dental structures covered with thermal insulation stickers.

The results of the research showed that the local temperature in the hard and soft tissues of the oral cavity in all the studied people in the conditions of room temperature before going out into the cold was normal and practically did not differ from each other. Then, after starting to inhale cold air with an open mouth, the local temperature of the hard and soft tissues began to decrease very quickly. Hard tissues were the fastest to cool. Of these, the most quickly and significantly cooled installed metal dental 
crowns and braces. Then, after starting to inhale cold air with an open mouth, the local temperature of the hard and soft tissues began to decrease very quickly. Hard tissues were the fastest to cool. Of these, the most rapidly and significantly cooled installed metal dental crowns and braces in those people who had them open and nothing covered. At the same time, these people felt an ache and pain in their cold teeth. After 10 minutes of being in the cold and inhaling cold air with an open mouth, the local temperature in the hard and soft tissues of the oral cavity was reduced by all of the researched volunteers. However, after removing the thermal insulation stickers from the dentition, it turned out that the temperature of the teeth and dental structures under them was much higher than that of the volunteers in all other groups. In addition, in the volunteers in all 3 subgroups with thermal insulation stickers were faster normalization of tissue temperature after returning them to the room and start breathing air at room temperature.

Therefore, thermal monitoring of the local temperature of teeth in the cold provides an assessment of the effectiveness of thermal insulation of teeth in real time. Therefore, the thermal imager can be used to create new materials and to develop new thermal insulation coatings for teeth, dental crowns, prostheses and braces for polar explorers, biathletes, military and mountain rescuers.

\section{Conclusion}

Thermal monitoring of local temperature and the use of stickers for thermal insulation of teeth with braces and dentures in the cold provides frost protection of gums, dental enamel and dentine against overcooling and frostbite, as well as prolonged performance of intensive performance of physical work at the limit of human capabilities, requiring air inhalation with open mouth.

\section{REFERENCES}

[1] Reshetnikov A, Kopylov M, Urakov A, Urakova T. Infrared diagnostics of the calluses disease from braces. 2016. ABSTRACTS. 13TH Quantitative Infrared Thermography. (July 4 - 8, 2016, Gdansk, Poland) Publishing by Gdansk University of Tecnology, 2016; $79-80$.

[2] Urakov AL, Urakova NA, Reshetnikov AP, Kopylov MV, Gabdrafikov RR. Thermal imaging diagnostics of blistering disease of cheeks and lips, which are caused by the brackets, and decorative sticker on the dentition with braces for prevention of this iatrogenic illness. Int J Gastroenterol Disord Ther. 2017; 4: 134.

[3] Reshetnikov AP, Reshetnikov YaA, Urakov AL. Decorative sticker for tooth heat insulation. RUS Patent № 2698349. Bull 2019; 24. 